\title{
Five Laboratory Tests Predict Patient Risk and Treatment Response in Hepatitis C: Veterans Affairs Data from 1999-2010
}

\author{
Ivy Tonnu-Mihara ${ }^{1}$, Tara Matsuda ${ }^{1,2}$, Jeff McCombs ${ }^{2, *}$, Sammy Saab $^{3}$, Patricia Hines ${ }^{4}$, Gilbert L'Italien ${ }^{4,5}$, \\ Timothy Juday, ${ }^{4,6}$, Yong Yuan ${ }^{4}$ \\ ${ }^{1}$ Veterans Affairs Long Beach Healthcare System, U.S. Department of Veterans Affairs, USA \\ ${ }^{2}$ Department of Pharmaceutical and Health Economics, School of Pharmacy, Leonard Schaeffer Center for Health Policy and Economics, \\ University of Southern California, USA \\ ${ }^{3}$ Department of Medicine and Surgery, David Geffen School of Medicine, University of California-Los Angeles, USA \\ ${ }^{4}$ Global Health Economics and Outcomes Research, Bristol-Myers Squibb, USA \\ ${ }^{5}$ Biogen Idec in Boston, USA \\ ${ }^{6}$ Abbvie Pharmaceuticals in Abbott Park, Il, USA
}

Copyright (C) 2016 by authors, all rights reserved. Authors agree that this article remains permanently open access under the terms of the Creative Commons Attribution License 4.0 International License

\begin{abstract}
Background and Aims: New Hepatitis C [HCV] drugs are expensive but highly effective but have created an overwhelming 'cash flow' problem for payers facing a large bolus of infected patients. Health plans are developing 'watchful waiting' strategies to safely defer treatment for low risk patients. This study identifies five laboratory tests which predict increasing risk of liver related events and documents that delaying treatment until after an abnormal lab test diminished effectiveness of treatment with interferon alpha plus ribavirin. Methods: Patients from the Veterans Administration's clinical registry of $\mathrm{HCV}$ patients [1999-2010] were screened for a detectable viral load at baseline and a recorded baseline genotype. The primary outcomes were time to death and time to time to a composite clinical event. Cox proportional hazards models were estimated with time dependent independent variables for initial treatment and first abnormal laboratory test. Results: 128,769 patients met all inclusion criteria. Abnormal values for five laboratory were associated with increased risk for the composite outcome/death: 1.35/1.84 for the AST/ALT ratio > $1 ; 2.35 / 5.01$ for albumin $<3 \mathrm{~g} / \mathrm{Dl} ; 1.58 / 1.15$ for GGT $>195$ IU/L; $3.85 / 1.55$ for platelet count $<100 \mathrm{k} / \mathrm{mm}^{2}$ and $4.48 / 2.39$ for alpha fetoprotein $>144 \mathrm{ng} / \mathrm{mL}$. Delaying drug therapy until after an abnormal lab test significantly reduced treatment effectiveness. Discussion: Five tests predict liver complications for HCV patients which can be used to develop "watchful waiting" protocols which monitor untreated $\mathrm{HCV}$ patients over time and defer access to expensive new drug regimens to those patients most at risk adverse liver-related events.
\end{abstract}

Keywords Watchful Waiting, Abnormal Lab Test, Risk Predictors, Delayed Treatment

\section{Introduction}

Hepatitis C (HCV) affects approximately 130-170 million people worldwide $[1,2]$ and an estimated 3.2 million people in the U.S.[3] HCV patients are at risk for developing progressive liver disease including cirrhosis, liver failure and hepatocellular carcinoma (HCC).[1, 2, 4-6] However, many patients with chronic HCV infections may fail to develop significant symptoms over their lifetime. Progression to cirrhosis is estimated to be about $5-20 \%$ after 20 years of infection.[7] It is estimated that $13.1 \%$ of patients with HCV in 2005 will die of liver-related causes by 2030 [8], increasing to $36.8 \%$ by 2060 .[9]

While HCV is considered cured if the patient achieves sustained viral response (SVR)[10], achieving SVR using older therapies [pegylated interferon-alpha and ribavirin] has been daunting. Standard therapy requires 24 to 48 weeks of weekly injections and is associated with significant side effect burden. Standard therapy achieves SVR rates ranging between $14-24 \%$ for $\mathrm{HCV}$ genotype 1 and $37-52 \%$ for genotypes 2 or 3.[11-13] More recent "triple" therapy, achieved by adding a protease inhibitor to pegylated interferon-alpha and ribavirin, is associated with a significant increase in SVR rates and an increase in frequency and severity of adverse side effects such as anemia, neutropenia, thrombocytopenia, rash, and gastrointestinal events.[14] Other reports suggest that "triple therapy" may increase SVR rates while reducing the side effects that limit tolerability.[15-21] It is not surprising that clinicians patients faced the twin problems of patient reluctance to initiate 
therapy and non-adherence once treatment is initiated.

The FDA has approved two new therapies [Fall 2014] which have radically changed the decision by patients and physicians when to initiate treatment by radically increasing expected SVR rates and shortening the duration of therapy. However, the costs of these therapies have created a need to ration drug using 'watchful waiting'/deferred treatment strategies under which clinicians and patients monitor the patient's risk of disease progression over time and initiate treatment before its effectiveness is adversely impacted.

The objective of this research is twofold. First, we use a large historical cohort of HCV patients from to document which specific abnormal laboratory tests predict an increased risk of liver-related liver events, including death. We then investigate whether or not a delay in initiating standard treatment until after one or more of these laboratory tests become abnormal degrades treatment effectiveness relative to 'early' treatment.

\section{Methods}

Data

The data used in this study were derived from the Veterans Administration [VA] clinical case registry (CCR) for HCV infected patients. Potential HCV patients were identified by the presence of an HCV-related ICD-9 diagnosis code or a positive viral load lab test. Local CCR coordinators then manually confirm or reject the patient for inclusion in the CCR and all historical data from the patient's electronic medical record (EMR) were added to the CCR. The VA EMR system was fully implemented in 1999 and provides data on patient demographic and clinical characteristics, diagnoses, laboratory test results and prescription and medical care utilization for over a decade.[22] Data period for this study cover 1999-2010.

A patient-level analytic database was created consisting of summary variables for each month before and after the patient's date of diagnosis [CCR enrollment date]. The post-index period for any individual patient could range up to 10 years depending on their index date. The following summary data were created:

1. Patient demographic data (age in months at baseline, gender, race, ethnicity, and geographic region at baseline).

2. Body mass index [BMI] in each month that these characteristics were recorded.

3. The patient's diagnostic profile consisting of monthly dichotomous variables reflecting the diagnoses recorded each month.

4. Monthly dichotomous variables for hospital admissions for any diagnosis and for liver related diagnoses.

5. Monthly values for most common laboratory tests, including viral load [VL] and viral genotype. Missing values were assigned when no tests were recorded during the month.

6. Prescription drug data were used to create a time-dependent dummy indicating whether or not the patient received HCV-related treatment before each event included as a study outcome.

\section{Sample Selection Criteria}

All patients were screened for a detectible HCV viral load [> $25 \mathrm{IU} / \mathrm{ml}]$ and a documented HCV viral genotype. The minimum value for a detectable viral load was selected based on the 'best' test methodology available within the VA system during the study period [1999-2010] and across multiple VA sites that reported data into the CCR. This selection criteria errored on the side of including patients whose viral load was reported as 'undetectable' at a higher minimum value [e.g., $<600 \mathrm{IU} / \mathrm{ml}$ ] using older testing methods.

\section{Primary and Secondary Outcomes}

Individuals infected with $\mathrm{HCV}$ are at risk for progressive liver disease and related complications such as cirrhosis, liver failure and hepatocellular carcinoma (HCC) which frequently require hospitalization and are at increased the risk of death.[1, 2, 4-6] The primary outcomes for this analysis are a composite of observed liver-related, clinical complications [compensated cirrhosis, decompensated cirrhosis, HCC or a liver-related hospitalization] or all-cause mortality. To ensure the clinical complications were due to chronic HCV infection, any composite clinical outcome occurring within a one-year "washout" period following the index date were not counted. The secondary outcomes included the individual elements of the clinical composite analyzed individually.

Monthly dichotomous variables were created for the outcomes of the study based on recorded diagnostic codes [e.g., diagnosis of cirrhosis, etc.] and selected CPT-4 codes included in data from hospital admission data and outpatient services. Liver-related hospitalization events were defined as the first occurrence of a diagnosis for complications due to $\mathrm{HCV}$ (see Appendix 1) as the primary diagnosis in inpatient visits. Compensated cirrhosis and HCC outcomes were compiled by searching the inpatient, outpatient and problem lists for matching ICD-9 codes [571.5, 571.2, 571.6 and 155,155.1, 155.2, respectively]. Decompensated cirrhosis was defined as a diagnosis of cirrhosis and a diagnosis of hepatic coma [70.44, 71.71, 348.3, 348.31, 572.2], portal hypertension [572.3], hepatorenal syndrome [572.4], jaundice [782.4], ascites [789.59], or esophageal varices $[456,456.1,456.2,456.21]$ or an FIB-4 score $>3.25 .{ }^{23} \mathrm{An}$ FIB-4 score is a function of age, aspartate transaminase [AST] level, platelet count and alanine transaminase [ALT]. An FIB-4> 3.25 has been correlated with a Metavir fibrosis stage of F3-F4 [23] or an Ishak fibrosis stage of F4-F6. [24] Vallet-Pichard, et al. also found the FIB-4 to have a positive predictive value to confirm the existence of significant fibrosis of $82.1 \%$ in a HCV infected cohort.[23]

\section{Statistical Methods}

The time-to-event variables for primary and secondary 
outcomes were analyzed using Cox proportional hazards.[25] Time-related independent variables were created for several risk factors to take advantage of the longitudinal nature of the data. The time-related laboratory test risk factors assessed whether or not the laboratory test values were abnormal immediately prior to the event date. The time-related treatment variable was included to assess whether or not treatment was initiated before or after the occurrence of each liver-related outcome variable. The time at which treatment is initiated is endogenous over time and correlated with observed and unobservable patient factors that are also correlated with patient outcomes. Therefore, the treatment effects measured in this analysis may be biased to the extent that important but unobserved patient characteristics are not included in the statistical models. However, the objective of this research is to test whether or not initiating treatment after one or more of the patient's laboratory value becomes abnormal rather than develop an unbiased estimate of treatment effects. While complicated, the methods used are at the heart of the analysis which ultimately provides information on what do clinicians watch to determine whether or not treatment is needed, and whether or not it is possible to wait too long, thus diminishing the effectiveness of treatment at reducing future risk. The answer to this question will be measured by the estimated coefficient for the interaction term between the time dependent dichotomous variables for treatment and the existence of one or more abnormal lab test.

A total of 22 individual clinical laboratory tests were entered into the analyses of each adverse liver event and death in order to determine which abnormal laboratory tests were predictive of these events. Laboratory test result data were entered as time-to-abnormal test result and the specific cut-points for defining an abnormal value can be found in Table 2. These cut-off values were set by the authors at a level high enough to generate wide agreement that the recorded lab value was abnormal.

All study patients were required to have a detectable viral load in order to focus the analysis on patients who could benefit from treatment. Separate Cox models were estimated for each primary and secondary outcome under study. Each analysis began by estimating a Cox model with all possible risk factors included in the list of independent variables, including the complete list of 22 common laboratory tests thought to be related to liver related adverse events. Since models of event risk should be parsimonious to be useful in clinical practice, the results for these all-inclusive models were reviewed and non-significant factors $[p>0.20]$ were dropped from subsequent model estimates. Age, gender, $\mathrm{HCV}$ genotype, and race were included in all models regardless of their statistical significance. Race and ethnicity were initially entered as separately coded categories. However, the significant correlation between race and ethnicity in this VA sample resulted in ethnicity being dropped from the final model specification. The dummy variables denoting a diabetes diagnosis at baseline and any hospital admission in the 6 months prior to the patient's index date were included in final model specifications based on statistical significance.

Once we documented which abnormal laboratory tests were predictive of future liver events, we tested if treatment effectiveness was dependent on the timing of treatment either before or after any one lab value becomes abnormal. This was accomplished by entering both the time dependent treatment variable and its interaction term with a time dependent variable denoting whether or not any abnormal laboratory test preceded treatment. This interaction variable measures the extent to which the effect of treatment initiated after an abnormal laboratory test is statistically different from the impact of 'early' treatment.

The VA restricted access to CCR data to VA employees [Dr. Tonnu-Mihara] and employees without compensation [Matsuda]. Authors from the funding source [Yuan, Juday, Hines and L'Italien] were involved the research design and with reviewing and interpreting statistical results. All authors reviewed and approved the final manuscript. The analysis was conducted using SAS v9.2.[26]

\section{Results}

\section{Descriptive Statistics}

Table 1. Patient Characteristics

\begin{tabular}{|c|c|c|}
\hline \multirow[b]{2}{*}{ Treatment Data } & \multicolumn{2}{|c|}{$\mathrm{N}=128,769$} \\
\hline & Count or Mean & $\%$ or $\mathrm{SD}$ \\
\hline Treated & 31,284 & $24.3 \%$ \\
\hline Untreated & 97,485 & $75.7 \%$ \\
\hline \multicolumn{3}{|l|}{$\begin{array}{c}\text { Achieved Undetectable Viral } \\
\text { Load }\end{array}$} \\
\hline Overall & 5,180 & $4.0 \%$ \\
\hline Under Treatment & 5,141 & $16.4 \%$ \\
\hline \multicolumn{3}{|l|}{ Patient Demographics } \\
\hline Gender [Male] & 124,980 & $97.1 \%$ \\
\hline Age [mean in years] & 51.8 & 6.9 \\
\hline \multicolumn{3}{|l|}{ Race } \\
\hline White & 66,168 & $51.4 \%$ \\
\hline Black & 40,239 & $31.3 \%$ \\
\hline Asian & 168 & $0.1 \%$ \\
\hline Other & 22,194 & $17.2 \%$ \\
\hline \multicolumn{3}{|l|}{ Ethnicity } \\
\hline Non-Hispanic & 107,586 & $83.6 \%$ \\
\hline Hispanic & 6,901 & $5.4 \%$ \\
\hline Other & 14,282 & $11.1 \%$ \\
\hline \multicolumn{3}{|l|}{ HCV Genotype } \\
\hline 1 & 102191 & $79.4 \%$ \\
\hline 2 & 15113 & $11.7 \%$ \\
\hline 3 & 9851 & $7.7 \%$ \\
\hline other & 1614 & $1.23 \%$ \\
\hline Pre-index Admission [6 months] & 20938 & $16.3 \%$ \\
\hline Diabetes at baseline & 15091 & $11.7 \%$ \\
\hline
\end{tabular}


A total of 128,769 patients met the study inclusion criteria. Only $24.3 \%$ of patients received treatment at any time following diagnosis [Table 1]. Only $16.4 \%$ of treated patients achieved an undetectable viral load post-treatment [ $4 \%$ of all patients]. The average post-index period consisted of 6.1 years [Standard deviation $(\mathrm{SD})=3$ ]. The $\mathrm{VA} / \mathrm{HCV}$ patients are predominately male of either white or black race [ $51.4 \%$ and $31.3 \%$ respectively]. The mean age is 52 years [SD $=6.9]$ and close to $80 \%$ of patients were genotype 1 , followed by $12 \%$ being genotype 2 . Over $16 \%$ of patients had a history of a hospital admission within 6 months prior to the index date and $12 \%$ of patients had a diabetes diagnosis at baseline.
Predictive Laboratory Tests

The laboratory data in Table 2 document the deteriorating health status of the study HCV population over time. The proportion of patients with abnormal values is higher in the post-index period for all laboratory tests with the exception of $\mathrm{HbAlc}$ for diabetes and for viral load. The laboratory tests found to be correlated with the risk of long-term liver complications and death are denoted by a bold font in Table 2.

The parsimonious models for each of our primary and secondary outcomes are displayed in Table 3 [Primary Outcomes] and Table 4 [Secondary Outcomes]. The 'side-by-side' arrangement of the models makes it easy to evaluate the extent to which risk factors are shared across target outcomes, and the relative strength of risk factors across outcomes.

Table 2. Laboratory Data

\begin{tabular}{|c|c|c|c|c|c|}
\hline \multirow[b]{2}{*}{ LABORATORY TESTS } & \multicolumn{2}{|c|}{ BASELINE } & \multirow[t]{2}{*}{ Definition of Abnormal } & \multicolumn{2}{|c|}{ POST-INDEX } \\
\hline & $\mathrm{N}$ & \% Abnormal & & $\%$ Abnormal & $\mathrm{N}$ \\
\hline BMI [Body Mass Index] & 125069 & 28.3 & $>35$ & 45.1 & 125069 \\
\hline Viral Load [IU/mL] & 128769 & 100 & $>25$ & 95.6 & 128769 \\
\hline \multicolumn{6}{|l|}{ Liver Function Tests } \\
\hline Direct bilirubin $[\mathrm{mg} / \mathrm{dL}]$ & 29536 & 0.9 & $>3.9$ & 3.3 & 89039 \\
\hline Albumin [g/dL] & 74619 & 7.3 & $<3.0$ & 24.0 & 126171 \\
\hline Alanine transaminase (ALT) $[\mathrm{IU} / \mathrm{L}]$ & 81410 & 21.9 & $>120$ & 40.2 & 127199 \\
\hline Aspartate transaminase (AST) $[\mathrm{IU} / \mathrm{L}]$ & 62808 & 15.8 & $>120$ & 32.5 & 105794 \\
\hline AST/ALT Ratio & 61221 & 32.1 & $>1$ & 67.3 & 105680 \\
\hline Alkaline phosphatase (ALP) [IU/L] & 80344 & 0.5 & $>345$ & 2.8 & 126787 \\
\hline Gamma-glutayltransferase (GGT) [IU/L] & 19514 & 25.2 & $>195$ & 30.5 & 64651 \\
\hline \multicolumn{6}{|l|}{ Hematology } \\
\hline Red Blood Cells(RBC) $\left[\mathrm{M} / \mathrm{mm}^{3}\right]$ & 56641 & 0.3 & $<2.19$ & 3.4 & 95537 \\
\hline Hemoglobin [g/dL] & 77935 & 1.0 & $<8$ & 7.8 & 126417 \\
\hline Hematocrit [\%] & 75210 & 1.3 & $<25$ & 9.7 & 126073 \\
\hline White Blood Cells(WBC) $\left[\mathrm{k} / \mathrm{mm}^{3}\right]$ & 77093 & 0.3 & $<2$ & 7.5 & 126246 \\
\hline Platelets $\left[\mathrm{k} / \mathrm{mm}^{3}\right]$ & 77565 & 7.9 & $<\mathbf{1 0 0}$ & 25.8 & 126253 \\
\hline Neutrophils & 53498 & 3.2 & $<30$ & 12.5 & 104403 \\
\hline \multicolumn{6}{|l|}{ Renal Function/Panel } \\
\hline Creatinine $[\mathrm{mg} / \mathrm{dL}]$ & 83066 & 6.3 & $>1.4$ & 24.2 & 126691 \\
\hline Blood urea nitrogen [BUN] [mg/dL] & 79585 & 0.4 & $>75$ & 3.6 & 123719 \\
\hline $\begin{array}{l}\text { Estimated glomerular filtration rate [eGFR] } \\
{\left[\mathrm{mL} / \mathrm{min} / 1.73 \mathrm{~m}^{2}\right]}\end{array}$ & 19431 & 10.1 & $<60$ & 25.2 & 106849 \\
\hline \multicolumn{6}{|l|}{ Ions and trace metals } \\
\hline Sodium $[\mathrm{mEq} / \mathrm{L}]$ & 81325 & 0.5 & $<125$ & 4.1 & 125302 \\
\hline Potassium $[\mathrm{mEq} / \mathrm{L}]$ & 80746 & 0.2 & $<2.7$ & 2.0 & 124943 \\
\hline Total Serum Iron (Iron)[mcg/dL] & 9606 & 26.8 & $>140$ & 36.1 & 89629 \\
\hline Total Iron Building Capacity [TIBC] [mcg/dL] & 7457 & 22.4 & $>400$ & 29.1 & 74193 \\
\hline Ferritin $[\mathrm{ng} / \mathrm{mL}]$ & 9384 & 36.5 & $>300$ & 41.3 & 88880 \\
\hline \multicolumn{6}{|l|}{ Other tests } \\
\hline Glycosylated hemoglobin [HbA1c (\%)] & 23505 & 30.6 & $>7$ & 26.4 & 87375 \\
\hline Alpha fetoprotein [ng/mL] & 7809 & 1.2 & $>144$ & 2.6 & 101623 \\
\hline
\end{tabular}


Table 3. Impact of Drug Therapy and Abnormal Laboratory Values on the Risk of Liver-Related Adverse Events and Death

\begin{tabular}{|c|c|c|c|}
\hline & \multicolumn{2}{|c|}{ Composite of Clinical Outcomes } & \multirow[t]{2}{*}{ Death } \\
\hline & All Events & One-Year Washout & \\
\hline & $\mathrm{N}=123,065$ & $\mathrm{~N}=106,947$ & $\mathrm{~N}=128,769$ \\
\hline \multirow[t]{2}{*}{ Number of Events [\%] } & $\begin{array}{l}35,253 \\
{[28.6 \%]}\end{array}$ & $\begin{array}{c}18,595 \\
{[17.4 \%]}\end{array}$ & $\begin{array}{c}15,458 \\
{[12.0 \%]}\end{array}$ \\
\hline & \multicolumn{3}{|c|}{ Estimated Coefficients, HAZARD RATIOS and [95\% Confidence Intervals] } \\
\hline DRUG THERAPY & $\begin{array}{c}\quad-0.08 \\
\text { H.R. }=0.92 \\
{[0.84-1.01]}\end{array}$ & $\begin{array}{c}-0.08 \\
\text { H.R. }=0.92 \\
{[0.84-1.01]}\end{array}$ & $\begin{array}{c}-0.20 \\
\text { H.R. }=0.82 * * * \\
{[0.75-0.90]}\end{array}$ \\
\hline \multicolumn{4}{|l|}{ LAB TESTS ${ }^{\mathrm{a}}$} \\
\hline Albumin $[<3 \mathrm{~g} / \mathrm{dL}]$ & $\begin{array}{c}\text { 0.85 H.R. }=2.35 * * * \\
{[2.08-2.66]}\end{array}$ & $\begin{array}{c}0.86 \\
\text { H.R. }=2.37 * * * \\
{[2.09-2.69]}\end{array}$ & $\begin{array}{c}1.16 \\
\text { H.R. }=5.01 * * * \\
{[4.58-5.48]}\end{array}$ \\
\hline AST/ALT Ratio [>1] & $\begin{array}{c}\text { 0.30 } \\
\text { H.R. }=1.35 * * * \\
{[1.25-1.46]}\end{array}$ & $\begin{array}{c}0.30 \\
\text { H.R. }=1.35 * * * \\
{[1.24-1.46]}\end{array}$ & $\begin{array}{c}0.61 \\
\text { H.R. }=1.84 * * * \\
{[1.68-2.01]}\end{array}$ \\
\hline Alkaline phosphatase [> $345 \mathrm{IU} / \mathrm{L}]$ & & & $\begin{array}{c}0.87 \\
\text { H.R. }=2.39 * * * \\
{[2.04-2.80]}\end{array}$ \\
\hline Gamma-glutayltransferase [> 195 IU/L] & $\begin{array}{c}0.46 \\
\text { H.R. }=1.58 * * * \\
{[1.44-1.72]}\end{array}$ & $\begin{array}{c}0.47 \\
\text { H.R. }=1.60 * * * \\
{[1.46-1.75]}\end{array}$ & $\begin{array}{c}0.14 \\
\text { H.R. }=1.15 * * \\
{[1.06-1.25]}\end{array}$ \\
\hline Hematocrit $[<25 \%]$ & & & $\begin{array}{c}0.75 \\
\text { H.R. }=2.11 * * * \\
{[1.89-2.35]}\end{array}$ \\
\hline Platelets $\left[<100 \mathrm{k} / \mathrm{mm}^{3}\right]$ & $\begin{array}{c}1.35 \\
\text { H.R. }=3.85 * * * \\
{[3.49-4.24]}\end{array}$ & $\begin{array}{c}1.35 \\
\text { H.R. }=3.85 * * * \\
{[3.49-4.26]}\end{array}$ & $\begin{array}{c}0.44 \\
\text { H.R. }=1.55 * * * \\
{[1.43-1.68]}\end{array}$ \\
\hline Creatinine $[>1.4 \mathrm{mg} / \mathrm{dL}]$ & & & $\begin{array}{c}0.70 \\
\text { H.R. }=2.02 * * * \\
{[1.86-2.20]}\end{array}$ \\
\hline $\begin{array}{l}\text { Estimated Glomerular Filtration Rate } \\
\qquad\left[<60 \mathrm{~mL} / \mathrm{min} / 1.73 \mathrm{~m}^{2}\right]\end{array}$ & $\begin{array}{l}\quad-0.15 \\
\text { H.R. }=0.86^{*} \\
{[0.76-0.99]}\end{array}$ & $\begin{array}{l}\quad-0.14 \\
\text { H.R. }=0.870^{*} \\
{[0.760-0.99]}\end{array}$ & \\
\hline Sodium $[<125 \mathrm{mEq} / \mathrm{L}]$ & & & $\begin{array}{c}0.92 \\
\text { H.R. }=2.51 * * * \\
{[2.16-2.91]}\end{array}$ \\
\hline Serum Iron $[>140 \mathrm{mcg} / \mathrm{dL}]$ & $\begin{array}{c}0.12 \\
\text { H.R. }=1.13 * * \\
{[1.04-1.23]}\end{array}$ & $\begin{array}{c}0.12 \\
\text { H.R. }=1.14^{* *} \\
{[1.05-1.25]}\end{array}$ & \\
\hline Ferritin $[>300 \mathrm{ng} / \mathrm{mL}]$ & & & $\begin{array}{c}0.11 \\
\text { H.R. }=1.12 * * \\
{[1.04-1.20]} \\
\end{array}$ \\
\hline Glycosylated hemoglobin [> 7\%] & & & $\begin{array}{c}\quad-0.16 \\
\text { H.R. }=0.85^{* *} \\
{[0.76-0.95]}\end{array}$ \\
\hline Alpha fetoprotein [> $144 \mathrm{ng} / \mathrm{mL}]$ & $\begin{array}{c}1.50 \\
\text { H.R. }=4.48 * * * \\
{[3.48-5.77]}\end{array}$ & $\begin{array}{c}1.44 \\
\text { H.R. }=4.24 * * * \\
{[3.27-5.52]}\end{array}$ & $\begin{array}{c}0.87 \\
\text { H.R. }=2.39 * * * \\
{[2.06-2.78]}\end{array}$ \\
\hline
\end{tabular}

a. Lab tests that were included in the initial model specifications as potential risk factors but which never achieved statistical significance in any risk prediction model include: hemoglobin, white blood cell count, potassium

$* \mathrm{p}<0.05 ; * \mathrm{p}<0.01 ; * * \mathrm{p}<0.0001$ 
Table 4. Impact of Abnormal Laboratory Values on the Risk of Adverse Events

\begin{tabular}{|c|c|c|c|c|}
\hline & Cirrhosis & Decompensated Cirrhosis & Liver-Related Hospitalization & Hepatocellular Carcinoma \\
\hline & $\mathrm{N}=123,988$ & $\mathrm{~N}=128,055$ & $\mathrm{~N}=128,769$ & $\mathrm{~N}=128,481$ \\
\hline \multirow[t]{2}{*}{ Number of Events [\%] } & $17,926[14.5 \%]$ & $\begin{array}{c}8,429 \\
{[6.6 \%]}\end{array}$ & $\begin{array}{c}28,730 \\
{[22.3 \%]}\end{array}$ & $\begin{array}{c}4,517 \\
{[3.5 \%]}\end{array}$ \\
\hline & \multicolumn{4}{|c|}{ Estimated Coefficients, HAZARD RATIOS and [95\% Confidence Intervals] } \\
\hline DRUG THERAPY & $\begin{array}{c}0.02 \\
\text { H.R. }=1.02 \\
{[0.90-1.15]}\end{array}$ & $\begin{array}{c}0.00 \\
\text { H.R. }=1.00 \\
{[0.91-1.10]}\end{array}$ & $\begin{array}{c}-0.21 \\
\text { H.R. }=\mathbf{0 . 8 1} * * * \\
{[\mathbf{0 . 7 6 - 0 . 8 7 ]}}\end{array}$ & $\begin{array}{c}-0.01 \\
\text { H.R. }=0.99 \\
{[0.87-1.12]}\end{array}$ \\
\hline \multicolumn{5}{|l|}{ LAB TESTS $^{\mathrm{a}}$} \\
\hline $\begin{array}{r}\text { Direct bilirubin } \\
{[>3.9 \mathrm{mg} / \mathrm{dL}]}\end{array}$ & & & $\begin{array}{c}0.41 \\
\text { H.R. }=1.51 * * \\
{[1.11-2.06]}\end{array}$ & \\
\hline Albumin $[<3 \mathrm{~g} / \mathrm{dL}]$ & $\begin{array}{c}1.05 \\
\text { H.R. }=2.86 * * * \\
{[2.46-3.34]}\end{array}$ & $\begin{array}{c}1.34 \\
\text { H.R. }=3.82 * * * \\
{[3.48-4.20]}\end{array}$ & $\begin{array}{c}1.03 \\
\text { H.R. }=2.79 * * * \\
{[2.58-3.03]}\end{array}$ & $\begin{array}{c}0.78 \\
\text { H.R. }=2.19 * * * \\
{[1.90-2.51]}\end{array}$ \\
\hline AST/ALT Ratio $[>1]$ & $\begin{array}{c}0.37 \\
\text { H.R. }=1.45^{* * *} \\
{[1.29-1.63]}\end{array}$ & $\begin{array}{c}0.96 \\
\text { H.R. }=2.60 * * * \\
{[2.34-2.88]}\end{array}$ & $\begin{array}{c}0.45 \\
\text { H.R. }=1.57 * * * \\
{[1.47-1.67]}\end{array}$ & $\begin{array}{c}0.64 \\
\text { H.R. }=1.90 * * * \\
{[1.67-2.17]}\end{array}$ \\
\hline $\begin{array}{c}\text { Alkaline phosphatase }[>345 \\
\text { IU/L] }\end{array}$ & & & $\begin{array}{c}0.39 \\
\text { H.R. }=1.48^{* *} \\
{[1.15-1.90]}\end{array}$ & $\begin{array}{c}0.65 \\
\text { H.R. }=1.92 * * \\
{[1.28-2.86]}\end{array}$ \\
\hline $\begin{array}{c}\text { Gamma-glutayltransferase } \\
{[>195 \mathrm{IU} / \mathrm{L}]}\end{array}$ & $\begin{array}{c}0.59 \\
\text { H.R. }=1.81 * * * \\
{[1.60-2.04]}\end{array}$ & $\begin{array}{c}0.38 \\
\text { H.R. }=1.46^{* * *} \\
{[1.33-1.59]}\end{array}$ & $\begin{array}{c}0.30 \\
\text { H.R. }=1.35 * * * \\
{[1.26-1.44]}\end{array}$ & $\begin{array}{c}\text { 0.36 } \\
\text { H.R. }=1.44 * * * \\
{[1.27-1.64]}\end{array}$ \\
\hline $\begin{array}{l}\text { Red blood cell count } \\
{\left[<2.19 \mathrm{M} / \mathrm{mm}^{3}\right]}\end{array}$ & $\begin{array}{c}-1.31 \\
\text { H.R. }=0.27 * \\
{[0.08-0.94]}\end{array}$ & & & \\
\hline Platelets $\left[<100 \mathrm{k} / \mathrm{mm}^{3}\right]$ & $\begin{array}{c}1.70 \\
\text { H.R. }=5.45^{* * *} \\
{[4.80-6.19]}\end{array}$ & $\begin{array}{c}1.55 \\
\text { H.R. }=4.71 * * * \\
{[4.29-5.17]}\end{array}$ & $\begin{array}{c}0.76 \\
\text { H.R. }=2.14 * * * \\
{[2.00-2.30]}\end{array}$ & $\begin{array}{c}1.10 \\
\text { H.R. }=3.01 \% * * \\
{[2.64-3.42]}\end{array}$ \\
\hline Neutrophils $[<30]$ & & $\begin{array}{c}\quad-0.27 \\
\text { H.R. }=0.76^{*} \\
{[0.58-0.99]}\end{array}$ & & \\
\hline $\begin{array}{c}\text { Serum Iron } \\
{[>140 \mathrm{mcg} / \mathrm{dL}]}\end{array}$ & $\begin{array}{c}0.26 \\
\text { H.R. }=1.30^{* * *} \\
{[1.15-1.46]}\end{array}$ & & & \\
\hline Ferritin $[>300 \mathrm{ng} / \mathrm{mL}]$ & $\begin{array}{c}0.16 \\
\text { H.R. }=1.17 * * \\
{[1.04-1.32]}\end{array}$ & & & $\begin{array}{c}0.30 \\
\text { H.R. }=1.35^{* * *} \\
{[1.20-1.52]}\end{array}$ \\
\hline $\begin{array}{l}\text { Total Iron Building Capacity } \\
{[>400 \mathrm{mcg} / \mathrm{dL}]}\end{array}$ & $\begin{array}{c}0.19 \\
\text { H.R. }=1.21^{* *} \\
{[1.07-1.38]}\end{array}$ & & & \\
\hline $\begin{array}{l}\text { Alpha fetoprotein [> } 144 \\
\text { ng/dL] }\end{array}$ & $\begin{array}{c}0.97 \\
\text { H.R. }=2.64 * * * \\
{[1.84-3.79]}\end{array}$ & $\begin{array}{c}1.55 \\
\text { H.R. }=4.71 * * * \\
{[3.99-5.57]}\end{array}$ & $\begin{array}{c}1.13 \\
\text { H.R. }=3.11 * * * \\
{[2.64-3.67]}\end{array}$ & $\begin{array}{c}2.88 \\
\text { H.R. }=17.87 * * * \\
{[14.94-21.36]}\end{array}$ \\
\hline $\begin{array}{l}\text { Estimated Glomerular } \\
\text { Filtration Rate } \\
{\left[<60 \mathrm{~mL} / \mathrm{min} / 1.73 \mathrm{~m}^{2}\right]}\end{array}$ & $\begin{array}{c}-0.27 \\
\text { H.R. }=0.76^{* *} \\
{[0.63-0.91]}\end{array}$ & & & \\
\hline $\begin{array}{l}\text { Blood Urea Nitrogen [ }>75 \\
\mathrm{mg} / \mathrm{dL}]\end{array}$ & & & & $\begin{array}{c}-1.90 \\
\text { H.R. }=0.15^{* *} \\
{[0.04-0.51]}\end{array}$ \\
\hline
\end{tabular}

a. Lab tests that were included in the initial model specifications as potential risk factors but which never achieved statistical significance in any risk prediction model include: hemoglobin, white blood cell count, potassium ${ }^{*} \mathrm{p}<0.05 ; * * \mathrm{p}<0.01 ; * * * \mathrm{p}<0.0001$

An abnormal AST/ALT ratio increased the risk of the composite of clinical liver events by approximately $35 \%$ [Hazard ratio (H.R.) $=1.35 ; 95 \%$ confidence interval $(\mathrm{CI})=(1.25-1.46)]$ and increased the risk of death by nearly $84 \% \quad[$ H.R. $=1.84 ; \mathrm{CI}=(1.68-2.01)]$. Abnormally low albumin increased the risk of long-term liver complications by $135 \%[$ H.R. $=2.35 ; \mathrm{CI}=(2.10-2.66)]$ and the risk of death five-fold $[$ H.R. $=5.01 ; \mathrm{CI}=(4.58-5.48)]$. Abnormal gamma-glutayltransferase [GGT] increased the risk of long-term liver complications by $58 \%[$ H.R. $=1.58 ; \mathrm{CI}=$ (1.44-1.72)] and death by $15 \%$ [H.R. $=1.15 ; \mathrm{C} \mathrm{I}=(1.06-1.25)]$ while abnormally low platelet levels increased the risk of liver events by nearly four-fold [H.R. $=3.85 ; \mathrm{CI}=(3.49-4.24)]$ and death by $55 \%[$ H.R. $=1.55 ; \mathrm{CI}=(1.43-1.68)]$. Finally, abnormal alpha fetoprotein increased the risk of liver-related clinical outcomes by nearly 5 -fold $[$ H.R. $=4.48 ; \mathrm{CI}=$ 
(3.48-5.77)] and more than doubled the risk of death [H.R. $=$ 2.39; $\mathrm{CI}=(2.06-2.78)]$.

All four clinical outcomes share common results for the five laboratory tests (ALT/AST ratio, albumin, GGT, alpha fetoprotein, platelets) that increased the risk of the composite clinical outcome and death [Table 4]. Several additional laboratory tests have crept into the individual models especially for cirrhosis, in which serum iron, ferritin and total iron binding capacity [TIBC] are predictive of higher risk, but an abnormal red blood cell count and blood urea nitrogen are predictive of lower risk. Other 'protective' abnormal lab values are blood urea nitrogen [BUN] for HCC and abnormal neutrophil counts for decompensated cirrhosis. Abnormally high alkaline phosphatase [ALP] is predictive of increased risk of liver-related hospitalizations and HCC.

\section{Delaying Treatment}

Estimates of the impact of initiating treatment before and after the emergence of an abnormal laboratory tests are presented in [Table 5]. The second column of Table 5 repeats the average results of drug therapy effectiveness found in Tables 3 and 4 to assist in interpreting results. The last two columns of Table 5 report our estimates of the effectiveness of drug therapy started 'early' [before any abnormal laboratory value is reported] or 'late' [following an abnormal laboratory test]. There are two tests of significance provided for the estimated effectiveness of 'late' treatment. The first measure of statistical significance denoted by '*' footnotes designates whether or not the estimated effect is different from the null hypothesis. The second statistical test, designated by ' $a$ ' indicates whether or not the estimated effects for early and late initiation of drug therapy are statistictically different. That is, did delaying the initiation of treatment adversely impact the effectiveness of initiating treatment?

There are two basic results of significance. First, the initiation of treatment prior to any laboratory test becoming abnormal is uniformly protective, but limited. Early treatment reduced the risk of the composite clinical event is $20 \%$ [H.R. $=0.80 ; \mathrm{CI}=(0.70-0.90]$ while the reduction in the risk of death is $22 \%$ [H.R. $=0.78 ; \mathrm{CI}=(0.65-0.93)]$. The limited impact of initiating treatment is not surprising given that only $16.4 \%$ of patients who initiate treatment achieve an undetectable viral load. Second, initiating standard HCV treatment after one or more laboratory test becomes abnormal never has a significant impact on the risk of these events. That is, delaying standard treatment until the patient exceeds any one of the lab test upper bounds listed in Table 2 significantly degrades the effectiveness of treatment.

Table 5. Impact of Treatment Initiation Before and After First Abnormal Lab Test

\begin{tabular}{|c|c|c|c|}
\hline Event & Overall & Before Abnormal Lab & After Abnormal Lab \\
\hline & \multicolumn{3}{|c|}{ Estimated Coefficients, HAZARD RATIOS and [95\% Confidence Intervals] } \\
\hline Cirrhosis & $\begin{array}{c}0.02 \\
\text { H.R. }=1.02 \\
{[0.90-1.15]}\end{array}$ & $\begin{array}{c}-0.19 \\
\text { H.R. }=0.83 * \\
{[0.69-0.99]}\end{array}$ & $\begin{array}{c}0.16 \\
\text { H.R. }=1.17^{\mathrm{a}} \\
{[0.95-1.74]}\end{array}$ \\
\hline Decompensated Cirrhosis & $\begin{array}{c}0.00 \\
\text { H.R. }=1.00 \\
{[0.91-1.10]}\end{array}$ & $\begin{array}{c}-0.36 \\
\text { H.R. }=0.70^{* * * *} \\
{[0.59-0.83]}\end{array}$ & $\begin{array}{c}0.14 \\
\text { H.R. }=1.15^{\mathrm{a}} \\
{[0.81-1.64]}\end{array}$ \\
\hline Liver Related Hospitalization & $\begin{array}{c}-0.21 \\
\text { H.R. }=0.81^{* * * *} \\
{[0.76-0.87]}\end{array}$ & $\begin{array}{c}-0.36 \\
\text { H.R. }=0.70^{* * * *} \\
{[0.63-0.77]}\end{array}$ & $\begin{array}{c}-0.11 \\
\text { H.R. }=0.90^{\mathrm{a}} \\
{[0.72-1.14]}\end{array}$ \\
\hline $\mathrm{HCC}$ & $\begin{array}{c}-0.01 \\
\text { H.R. }=0.99 \\
{[0.87-1.12]}\end{array}$ & $\begin{array}{c}-0.37 \\
\text { H.R. }=0.69 * * \\
{[0.53-0.90]}\end{array}$ & $\begin{array}{c}0.08 \\
\text { H.R. }=1.08^{\mathrm{a}} \\
{[0.63-1.88]}\end{array}$ \\
\hline Composite Event & $\begin{array}{c}-0.08 \\
\text { H.R. }=0.92 \\
{[0.84-1.01]}\end{array}$ & $\begin{array}{c}-0.22 \\
\text { H.R. }=0.80^{* *} \\
{[0.70-0.92]}\end{array}$ & $\begin{array}{c}-0.04 \\
\text { H.R. }=0.96^{\mathrm{a}} \\
{[0.72-1.28]}\end{array}$ \\
\hline Death & $\begin{array}{c}-0.20 \\
\text { H.R. }=0.82 * * * \\
{[0.75-0.90]}\end{array}$ & $\begin{array}{c}-0.25 \\
\text { H.R. }=0.78^{* *} \\
{[0.65-0.93]}\end{array}$ & $\begin{array}{c}-0.19 \\
\text { H.R. }=0.83 \\
{[0.57-1.20]}\end{array}$ \\
\hline
\end{tabular}

${ }^{*} \mathrm{p}<0.05 ; * * \mathrm{p}<0.01 ; * * * \mathrm{p}<0.0001$

${ }^{a}$ Indicates a significant reduction in treatment effectiveness if treatment is initiated after the patient's first recorded abnormal laboratory test for albumin, AST/ALT ratio, platelets, GGT or alpha fetoprotein. 


\section{Discussion}

Tools are needed with which to manage newly approved drug therapies. Our results also suggest that delaying therapy until the patient's lab profile becomes 'abnormal' could compromise patient outcomes. Our results demonstrating the limited effectiveness of standard therapy are also consistent with earlier research using VA data [11] and other 'real world' treatment settings.[12, 13] Treatment rates in our VA sample using standard therapy are only $24.3 \%$ and few treated patients [16.4\%] achieved at least one undetectable level of viral load test $[4 \%$ of the total HCV study population]. It is no surprise that asymptomatic $\mathrm{HCV}$ patients have gambled in the past that they will not develop $\mathrm{HCV}$ complications in order to avoid the treatment burden associated with standard therapy.

New treatment options have reversed the dynamics of the treatment decision. Patients are now demanding treatment with expensive, efficacious and less burdensome therapies while payers seek to ration treatment to high risk patients. A cost-containment strategy of reserving treatment for patients 'abnormal' lab tests cannot be implemented because the thresholds used here to define abnormal lab tests were set high to avoid controversy about whether or not the test was truly abnormal. More research is needed to set the screening values for each of the 5 test in this analysis at a level high enough to predict increased event risk but low enough to avoid inappropriate delays in treatment that impact the effectiveness of treatment.

Previous research has found similar relationships between the risk of liver events and abnormal laboratory test results based on relatively small samples of patients with disease progression. Lee, et al. [27] found that elevated ALT levels were associated with an increased risk of HCC in Taiwan. $\mathrm{Hu}$, et al.[28] found that elevated AST and alpha-feta protein [AFP] were associated with stages 3-4 fibrosis in Taiwan patients. In a Japanese study of 459 patients, Imazeki, et al. [29] found that increasing fibrotic stage was highly correlated with the risk of all-cause mortality, but baseline ALT, AST, albumin and platelet counts were not statistically significant when fibrosis stage was entered as an explanatory variable. In a second small Japanese study [ $n=345]$, Shiratori, et al. [30] found elevated albumin to be protective for HCC and elevated AST to increase the risk of death. In a small study in Spain [N=568]. Fernandez-Rodriguez, et al. [31] found that baseline albumin $[<3.9 \mathrm{~g}$ per $100 \mathrm{ml}]$ was associated with a three-fold increase in the risk of long-term liver complications. In a study of treated patients, van der Meer, et al. found that an increasing AST/ALT ratio was associated with increased risk of mortality.[32] A study that used VA data found that abnormal laboratory values for albumin, AST, AST/ALT ratio, creatinine clearance, and sodium were associated with mortality in genotype 1 patients.[33] Finally, the HALT-C study found that changes from baseline for platelets, AST/ALT ratio, bilirubin and albumin were predictive of decompensated cirrhosis and liver-related death or liver transplantation.[34]
There are several additional and important limitations in our study. First, most HCV cohorts from the US would not match well with our study sample which consist almost exclusively of men. Second, treatment for HCV patients in the VA health care system may vary from treatment patterns found in the civilian health care system. However, the Veteran Administration is the largest provider of care to chronically HCV-infected patients in the US. [35]

The asymptomatic nature of $\mathrm{HCV}$ and the retrospective design of this study make it likely that the index date identified here will not correspond to the actual date of $\mathrm{HCV}$ infection. However, this uncertainty regarding the actual date of infection is common in clinical practice. Most patients with hepatitis $\mathrm{C}$ are unaware of their infection until their exposure to $\mathrm{HCV}$ is detected by routine screening of blood donations or their health status deteriorates due to the infection.

The changing sensitivity of HCV viral load tests also presented a challenge in defining an 'undetectable' viral load. While many older tests have a lower threshold of 600, newer tests are sensitive up to $10 \mathrm{IU} / \mathrm{mL}$. We chose to apply a cutoff of 25 as the limit of 'detectable' for all patients to be consistent, but it is highly likely that some patients with older labs will be mis-categorized due to the low sensitivity of the tests in use at that time.

Finally, our study does not capture medical care outside the VA system, such as the Medicare program. If patients received treatment for complications of liver disease outside the VA, these data may not have been captured and time to event data will be accurate or missing. However, we believe that most of the care for HCV patients was provided within VA system due to the complete coverage for prescription medications and the very high cost of $\mathrm{HCV}$ related medications.

\section{Abbreviations}

HCV: Hepatitis $\mathrm{C}$ virus

AST: Aspartate transaminase

ALT: Alanine transaminase

GGT: Gamma-glutayltransferase

HCC: Hepatocellular carcinoma

SVR: Sustained viral response

FDA: Food and Drug Administration

VA: Veterans Administration

CCR: Clinical case registry

ICD-9: International Classification of Diseases, $9^{\text {th }}$ edition

EMR: Electronic medical records

BMI: Body mass index

VL: viral load

CPT-4: Current Procedure Terminology, $4^{\text {th }}$ edition

FIB-4: Fibrosis-4 score

SD: Standard deviation

HbA1c: Glycated hemaglobin

H.R.: Hazard ratio

C.I.: Confidence interval 
AFP: Alpha fetoprotein

TIBC: Total iron building capacity

BUN: Blood urea nitrogen

ALP: Alkaline phosphatase

HIV: Human Immunodeficiency Virus

RBC: Red Blood Cells

WBC: White Blood Cells

eGFR: Estimated glomerular filtration rate

\section{Acknowledgements}

The authors want to acknowledge Anupama Kalsekar, MS from Bristol Myers Squibb for her scientific critique and Tim Morgan, M.D., from the VA in Long Beach for his support of the project.

Appendix. List of diagnoses for liver-related hospitalization outcome

\section{Liver-related diagnoses}

Acute or unspecified hepatitis $\mathrm{C}$ with hepatic coma

Chronic hepatitis $\mathrm{C}$ with hepatic coma

Other specified viral hepatitis with hepatic coma

Other specified viral hepatitis without mention of hepatic coma code range

Unspecified viral hepatitis with hepatic coma

Unspecified viral hepatitis $\mathrm{C}$ code range

Unspecified viral hepatitis without mention of hepatic coma

Toxoplasma hepatitis

Malignant neoplasm of liver, primary

Malignant neoplasm of intrahepatic bile ducts

Malignant neoplasm of liver, not specified as primary or secondary

Esophageal varices with bleeding

Spontaneous bacterial peritonitis

Alcoholic fatty liver

Acute alcoholic hepatitis,

Alcoholic cirrhosis of liver

Alcoholic liver damage, unspecified

Chronic hepatitis, unspecified

Chronic persistent hepatitis

Chronic active hepatitis

Cirrhosis of liver without mention of alcohol

Biliary cirrhosis (chronic nonsuppurative destructive cholangitis)

Other chronic non-alcoholic liver disease

Unspecified chronic liver disease without mention of alcohol

Portial pyemia

Hepatic coma

Portal hypertension

Hepatorenal syndrome

Other sequelae of chronic liver disease

Hepatitis in viral diseases classified elsewhere

Hepatitis in other infectious diseases classified elsewhere.

Hepatitis, unspecified (trauma and toxic reactions)

Other specified disorders of liver

Unspecified disorder of liver

Jaundice

Hepatomegaly

Ascites

Hepatitis C carrier, unspecified

Liver transplant status 


\section{REFERENCES}

[1] Lavanchy D: The global burden of hepatitis C. Liver Int 2009, 29(Suppl 1):74-81.

[2] Chen SL, Morgan TR: The natural history of hepatitis C virus (HCV) infection. Int J Med Sci 2006, 3(2):47-52.

[3] Armstrong GL, Wasley A, Simard EP, McQuillan GM, Kuhnert WL, Alter MJ: The prevalence of hepatitis C virus infection in the United States, 1999 through 2002. Ann Intern Med 2006, 144(10):705-714.

[4] Seeff LB: The history of the "natural history" of hepatitis C (1968-2009). Liver Int 2009, 29(Suppl 1):89-99.

[5] Klevens RM, Hu DJ, Jiles R, Holmberg SD: Evolving epidemiology of hepatitis $\mathrm{C}$ virus in the United States. Clin Infect Dis 2012, 55(Suppl 1):S3-9.

[6] Smith BD, Morgan RL, Beckett GA, Falck-Ytter Y, Holtzman D, Teo CG, et al: Recommendations for the identification of chronic hepatitis $\mathrm{C}$ virus infection among persons born during 1945-1965. MMWR Recomm Rep 2012, 61(RR-4):1-32.

[7] CDC. Hepatitis C Information for Health Professionals. Available from: http://www.cdc.gov/hepatitis/HCV/HCVfaq.htm\#section1. Accessed October 31, 2014.

[8] Davis GL, Albright JE, Cook SF, Rosenberg DM: Projecting future complications of chronic hepatitis $\mathrm{C}$ in the United States. Liver Transpl 2003, 9(4):331-338.

[9] Rein DB, Wittenborn JS, Weinbaum CM, Sabin M, Smith BD, Lesesne SB: Forecasting the morbidity and mortality associated with prevalent cases of pre-cirrhotic chronic hepatitis C in the United States. Dig Liver Dis 2011, 43(1):66-72.

[10] $\mathrm{Ng} \mathrm{V}$, Saab S. Effects of a sustained virologic response on outcomes of patients with chronic hepatitis C. Clin. Gastroenterol. Hepatol. 2011, 9(11):923-30)

[11] Kramer JR, Kanwal F, Richardson P, Mei M, El-Serag HB: Gaps in the achievement of effectiveness of HCV treatment in national VA practice. J Hepatol 2012, 56(2):320-325.

[12] Shepherd J, Brodin H, Cave C, Waugh N, Price A, Gabbay J. Pegylated interferon alpha- $2 a$ and $-2 b$ in combination with ribavirin in the treatment of chronic hepatitis $\mathrm{C}$ : a systematic review and economic evaluation. Technol Assess. 2004;8(39):iii-iv, 1-125.

[13] Borroni G, Andreoletti M, Casiraghi MA, Ceriani R, Guerzoni P, Omazzi B, et al. Effectiveness of pegylated interferon/ribavirin combination in 'real world' patients with chronic hepatitis $\mathrm{C}$ virus infection. Aliment Pharmacol Ther. 2008;27(9):790-7.

[14] Gaetano JN, Reau N. Hepatitis C: management of side effects in the era of direct-acting antivirals. Curr Gastroenterol Rep. 2013 15(1):305. doi: 10.1007/s11894-012-0305-1.

[15] McHutchison JG, Everson GT, Gordon SC, Jacobson IM, Sulkowsi M, Kauffman R, et al. Telaprevir with peginterferon and ribavirin for chronic HCV genotype 1 infection. N Engl J Med 2009;360:1827-38.

[16] Poordad F, McCone J, Bacon BR, Bruno S, Manna MP, Sulkowski MS, et al. Boceprevir for Untreated Chronic HCV Genotype 1 Infection. N Eng J Med 2011;364(13):1195-206.

[17] Bacon BR, Gordon SC, Lawitz E, Marcellin P, Vierling JM, Zeuzem S, et al. Boceprevir for Previously Treated Chronic $\mathrm{HCV}$ Genotype 1 Infection $\mathrm{N}$ Eng $\mathrm{J}$ Med 2011;364(13):1207-17.

[18] Jacobson IM, McHutchison JG., Dusheiko G, Di Bisceglie AM, Reddy KR, Bzowei NH, et al. Telaprevir for Previously Untreated Chronic Hepatitis C Virus Infection. N Engl J Med 2011;364(25):2405

[19] Zeuzem S, Andreone P, Pol S, Lawitz, E, Diago, M, Roberts, $\mathrm{S}$, et al. Telaprevir for Retreatment of HCV Infection. N Eng J Med 2011;364(25):2417-28

[20] Jesudian AB, de Jong YP, Jacobson IM. Emerging therapeutic targets for hepatitis $\mathrm{C}$ virus infection. Clin Gastroenterol Hepatol. 2013 Jun;11(6):612-9.

[21] Doyle JS, Aspinall E, Liew D, Thompson AJ, Hellard ME. Current and emerging antiviral treatments for hepatitis $\mathrm{C}$ infection. Br J Clin Pharmacol. 2013 Apr;75(4):931-43.

[22] Backus LI, Gavrilov S, Loomis TP, Halloran JP, Phillips BR, Belperio PS, et al. Clinical Case Registries: simultaneous local and national disease registries for population quality management. J Am Med Inform Assoc 2009, 16(6):775-783.

[23] Vallet-Pichard A, Mallet V, Nalpas B, Verkarre V, Nalpas A, Dhalluin-Venier V, et al., FIB-4: an inexpensive and accurate marker of fibrosis in HCV infection. Comparison with liver biopsy and fibrotest. Hepatology 2007, 46(1):32-36.

[24] Sterling RK, Lissen E, Clumeck N, Sola R, Correa MC, Montaner J, et al. Development of a simple noninvasive index to predict significant fibrosis in patients with $\mathrm{HIV} / \mathrm{HCV}$ coinfection. Hepatology 2006;43:1317-1325.

[25] Cox DR. Regression Models and Life-Tables. Journal of the Royal Statistical Society. Series B (Methodological). 1972;34(2):187-220.

[26] Allison, PD. (2010). Survival analysis using SAS: A practical guide (2nd Edition). Cary, NC, USA: SAS Institute Inc.

[27] Lee MH, Yang HI, Lu SN, Jen CL, Yeh SH, Liu CJ, Chen PJ, You SL, Wang LY, Chen WJ et al: Hepatitis C virus seromarkers and subsequent risk of hepatocellular carcinoma: long-term predictors from a community-based cohort study. J Clin Oncol 2010, 28(30):4587-4593.

[28] $\mathrm{Hu}$ SX, Kyulo NL, Xia VW, Hillebrand DJ, Hu KQ. Factors associated with hepatic fibrosis in patients with chronic hepatitis C: a retrospective study of a large cohort of US patients. J Clin Gastroenterol 2009, 43(8):758-764.

[29] Imazeki F, Yokosuka O, Fukai K and Saisho H. Favorable prognosis of chronic hepatitis $\mathrm{C}$ after interferon therapy by long-term cohort study. Hepatology 2003;38(8):493-502.

[30] Shiratori Y, Ito Y, Yokosuka O, Imazeki, F, Nakata, R, Tanaka, N, et al. Antiviral therapy for cirrhotic hepatitis C: Association with reduced hepatocellular carcinoma development and improved survival. Ann Inter Med 2005;142(2):105-114. 
[31] Fernandez-Rodriguez CM, Alonso S, Martinez SM, Forns X, Sanchez-Tapias JM, Rincón D, et al. Peginterferon plus ribavirin and sustained virological response in HCV-related cirrhosis: Outcomes and factors prediction response. Am J Gastroenterol 2010;105(10):2164-2172.

[32] van der Meer AJ, Veldt BJ, Feld JJ, Wedemeyer H, Dufour JF, Lammert F, et al. Association between sustained virological response and all-cause mortality among patients with chronic hepatitis C and advanced hepatic fibrosis. JAMA 2012, 308(24):2584-2593.

[33] Backus LI, Boothroyd DB, Phillips BR, Belperio P, Halloran J, Mole LA. A sustained virologic response reduces risk of all-cause mortality in patients with hepatitis C. Clin Gastroenterol Hepatol. 2011;9(6):509-516.

[34] Ghany MG, Kim HY, Stoddard A, Wright EC, Seeff LB, Lok AS. Predicting clinical outcomes using baseline and follow-up laboratory data from the hepatitis C long-term treatment against cirrhosis trial. Hepatology. 2011;54(5):1527-1537

[35] Center for Quality Management in Public Health. The state of care for veterans with chronic Hepatitis C. In. Palo Alto, California: US Department of Veteran Affairs, Public Health Strategic Health Care Group, Center for Quality Management in Public Health; 2010. 